www. revis tad y o. com

\title{
Áreas clave para la alineación de la agilidad y resiliencia con la perspec- tiva de género en las organizaciones
}

Key areas for the alignment of agility and resilience with the gender perspective in organizations

\author{
Cuenca L. ${ }^{1}$, Navarro-Astor E. ${ }^{2}$, Alemany $\mathrm{MME}^{3}$, Boza A. ${ }^{4}$ \\ 1,3,4 Centro de Investigación en Gestión e Ingeniería de la Producción. Universitat Politécnica de València. Camino de Vera S/n. 46022, Valencia. \\ $\{$ llcuenca, mareva, aboza\}@cigip.upv.es \\ ${ }^{2}$ Escuela Técnica Superior de Ingeniería de Edificación, Dpto. Organización de empresas. Universitat Po-litècnica de València. Camino de Vera \\ S/n. 46022, Valencia. enavarro@omp.upv.es
}

Fecha de recepción: 06/07/2017

Fecha de aceptación: 08/11/2017

Resumen: La equidad de género en las organizaciones es un aspecto cada vez más necesario y demandado por la legislación; implica satisfacer el derecho constitucional de la igualdad. Hasta ahora, en su estudio, no se ha tenido en cuenta aspectos como agilidad y resiliencia. El presente artículo propone la definición preliminar de un modelo de madurez que permita evaluar la incorporación de la perspectiva de género sin limitar la agilidad y resiliencia en la organización, extrayendo la relación que indique qué aspectos de agilidad y resiliencia pueden verse afectados por las áreas donde la perspectiva de género debe ser incorporada.

Palabras clave: Perspectiva de género, Agilidad, Resiliencia, Modelo de madurez

Abstract: Ensuring gender equality in organizations is an increasingly necessary and demanded aspect of legislation; implies satisfying the constitutional right of equality. Has-ta now, in his study, has not taken into account variants that can affect the agility and resilience of the organization. The present article proposes the preliminary definition of a maturity model that allows to evaluate the incorporation of the gender perspective without limiting the agility and resilience in the organization, extracting the relation that indicates which aspects of agility and resilience can be affected by the areas where the gender perspective should be incorporated. It is proposed in this article to extend the alignment beyond information systems and technologies, incorporating aspects such as agility, resilience and gender perspective in supply chains. In this sense, the alignment will refer to the degree to which incorporating the gender perspective into the design of supply chains favors and drives agility and resilience, and how agile and resilient chains facilitate the incorporation of perspectives of gender in the design of the chain. On one hand, key areas have been identified as follows: Government and values, Work environment, Supply chain, Community, Consumers and Investment, and the general levels of maturity to be achieved. On the other hand, it has been defined the aspects to be taken into account to ensure their agility and resilience and the relationships with the key areas. The key area of government and values involves establishing the principles guiding considerations on gender equality in policies that determine organizational culture. These principles should be extended to all areas included as important to ensure the agility and resilience of the organization. Work environment corresponds to the definition of equal opportunities for women and men, eliminating obstacles that limit their participation. What will be related to the definition of facilities and resources, processes, people and strategy, which favors agility and resilience. The supply chain corresponds to new employment opportunities for women, both as employees and entrepreneurs. Assessing gender performance throughout the supply chain can be an important part of organizations' risk management and primarily affects processes, people and strategies. The community and consumers have an impact on processes, people and strategy, as they seek to strengthen efforts to promote gender equality in the organization, implement gender awareness in customer service, and analyze the feedback from clients, as well as, develop a more inclusive group of contracting within the community. Finally, with respect to the key investment area, it focuses mainly on the decisions of the investors that are frequently taken based on aspects of interest of their performance with regard to gender, which corresponds with the areas of people and strate-gy 


\section{Introducción}

De acuerdo al informe (Cinco Días 2016), dos de cada cinco empresas que han sufrido una situación no prevista que afectara al desarrollo normal de su actividad, como un ataque informático, un desastre natural, un incendio en las instalaciones, o cualquier otra situación que impacte en las actividades clave de la organización, no vuelven a abrir transcurridos los tres años. Además, una de cada dos sale del negocio después de los dos años.

En muchos casos, los sistemas de ayuda a la toma de decisiones no están preparados para reaccionar a cambios en el entorno de la cadena de suministro (CS) (Boza et al. 2014). Por otra parte, en la CS destaca la importancia de crear fuertes vínculos y relaciones. Las relaciones que se establecen en las cadenas de suministro, cuando son más diversas resultan más ricas, tienen en cuenta más aspectos, son más integradoras, con menos confrontación y agresividad. Esta diversidad se incrementa al garantizar la equidad de género en las organizaciones, aumentando así su agilidad y resiliencia, ya que puede mejorar la capacidad de reacción. En la gestión de la CS la cuestión de género también es relevante. Una investigación de Oxfam, sobre la situación de las mujeres en la CS del cacao en cuatro países, nos muestra el abandono, la desigualdad y el trato injusto al que hacen frente estas mujeres (Oxfam 2013). La gestión tradicional de la CS se ha ampliado para considerar factores como la agilidad y resiliencia que permitan hacer frente a un entorno cambiante, y al mismo tiempo, las nuevas redes de suministro, han creado nuevas oportunidades de empleo para ambos géneros. Monitorizar el desempeño de género en toda la CS puede constituir una parte importante de la gestión de riesgo de las organizaciones, por ejemplo, al ayudar a evitar daños a su reputación mediante la asociación a condiciones de trabajo discriminatorias y precarias.

\section{Perspectiva de género}

Las empresas son cada vez más conscientes de la necesidad de incorporar la perspectiva de género en su gestión y en su actividad diaria, pero carecen de herramientas para saber si estimulan correctamente la igualdad de género, si miden sistemáticamente su avance, o si se han identificado los indicadores adecuados que este análisis debe considerar (Miles 2011).

Conviene señalar la diferencia que existe entre los conceptos de igualdad, equidad y perspectiva de género. Existen muchas definicione que dependen del contexto en el que se usen, es necesario antes de abordar este tema, que queden claro las diferencias que existen entre cada concepto. Se ha tomado como referencia, las definiciones propuestas por la ONU Mujeres, en el documento Principios para el empoderamiento de las mujeres, quedando definidas de la siguiente manera

- Igualdad de género: Hace referencia al concepto por el que los hombres y las mujeres desarrollan sus capacidades personales y sus decisiones de forma libre, sin ningún tipo de limitación de género o prejuicios. Implica que todos los comportamientos, aspiraciones y necesidades inherentes a los hombres y mujeres se consideren, valoren y repartan equitativamente, y que no dependa del hecho de que hayan nacido hombre o mujer.

- Equidad de género: Se refiere a la justicia en el tratamiento de hombres y mujeres, según sus necesidades. Se deben incluir tratamientos considerados equivalentes en términos de derechos, beneficios, obligaciones y oportunidades.

- Perspectiva de género: Enfoque que permite evaluar como las políticas, programas, proyectos y actividades influyen en los hombres y las mujeres. Tiene en cuenta los roles basados en el género, las relaciones, los recursos, las necesidades sociales/económicas y otras limitaciones impuestas por la sociedad, la cultura o la etnia.

Incorporar la perspectiva de género implica incluir no solo dimensiones vinculadas directamente a los eslabones de la CS, sino también el estudio de las relaciones que se dan fuera de éstos (relaciones de género). Incluir el género en el análisis de una CS implica profundizar en los roles desempeñados por hombres y mujeres a lo largo de la cadena y en sus condiciones de trabajo, no solo con un fin descriptivo sino para comprender los procesos socioculturales, económicos e institucionales que llevan a su exclusión o inclusión en los diferentes eslabones (Alesina, 2007). Incorporar un análisis de género permitirá formular sistemas de objetivos y enfoques metodológicos con sensibilidad de género a partir de los resultados obtenidos. Muchos autores define la sensibilidad de género en términos de igualdad de remuneración y trato justo como un asunto social a lo largo de la CS (Prieto-Carron 2008). La perspectiva de género debe integrarse desde el enfoque metodológico y el sistema de objetivos (Claire Aanes et al. 2013). Se dice que la equidad de género se ha alcanzado cuando hombres y mujeres pueden acceder y disfrutar de las mismas recompensas, recursos y oportunidades. El objetivo es lograr resultados iguales en términos generales entre mujeres y hombres, y no exactamente el mismo resultado para todos los individuos (Australian Goverment 2016). El 
potencial de la igualdad de género se puede encontrar en diferentes países y sectores (Caven, Navarro-Astor, and Diop 2016), y en la mayor parte de los casos, la segregación es reconocida como uno de los principales factores que propician desigualdades entre mujeres y hombres en el mercado de trabajo. De acuerdo a (GRI \& IFC 2009) el análisis de la perspectiva de género se debe incluir en las siguientes categorías: Gobierno y Valores Organizacionales, Igualdad de Géneros en el Ambiente de Trabajo, Igualdad de Géneros y la CS, Igualdad de Géneros y la Comunidad, Igualdad de Géneros y los Consumidores, Igualdad de Géneros e Inversión.

\section{Agilidad y Resiliencia}

Respecto a la resiliencia de la CS podemos definirla como la capacidad de volver al estado original o deseado después de una perturbación importante (Sanchis and Poler 2011; 2014). La resiliencia es una característica obligatoria de la CS con el fin de proporcionar la capacidad de adaptarse a los cambios y desarrollarse en el largo plazo. Las organizaciones luchan por sobrevivir de las recesiones y las interrupciones, pero aquél más rápido en adaptarse no sólo sobrevive, sino que puede también dominar a sus competidores (Soni and Jain 2011). Conviene por tanto no separar la resiliencia de la agilidad. La agilidad de la CS se define generalmente como la capacidad de responder a los cambios imprevistos (Sheffi 2004). Puede definirse como el estado o la capacidad de moverse rápida y fácilmente. En consecuencia, una empresa ágil puede ajustarse a cualquier cambio inesperado o repentino en el ambiente, tanto rápida como eficientemente. La agilidad y resiliencia deben ser aplicadas a distintas áreas como son: Instalaciones y recursos, tecnologías, datos, aplicaciones, procesos, personas y estrategia (Bhamidipaty, Lotlikar, and Banavar 2007). Hasta la fecha, la perspectiva de género no se ha tenido en cuenta en el estudio de las variantes del análisis de resiliencia que abordan la dinámica social. A pesar de que el análisis de género y el análisis de resiliencia se refieren esencialmente a la comprensión del cambio en diversas formas, tienen entendimientos diferentes sobre cómo ocurre el cambio y cómo podemos entenderlo.

\section{Alineación y evaluación de la Perspectiva de Género con la Agilidad y Resiliencia.}

La alineación, en el contexto de los sistemas y tecnología de información (SI/TI), es el grado en que la estrategia de SI/TI favorece e impulsa la estrate- gia de negocio y viceversa (Cuenca, Boza, \& Ortiz, $2011,2015)$. Desde hace varios años, la alineación de negocios y de SI/TI es considerada por los ejecutivos de TI como uno de sus temas clave (Luftman 2003). Entre los beneficios de una buena alineación de negocio y TI están (Avison et al. 2004) (Haes and Grembergen 2004): 1) maximizar el valor de retorno de las inversiones en TI, 2) mejorar la posición competitiva a través de los sistemas de información, 3) proporcionar dirección y flexibilidad para reaccionar a los cambios. Este último beneficio está directamente relacionado con la agilidad y la resiliencia.

Se propone en este artículo extender la alineación más allá de los sistemas y tecnologías de la información, incorporando aspectos como la agilidad, resiliencia y perspectiva de género en cadenas de suministro. En este sentido la alineación hará referencia al grado en que el incorporar la perspectiva de género en el diseño de cadenas de suministro favorece e impulsa la agilidad y resiliencia, y cómo cadenas ágiles y resilientes facilitan la incorporación de la perspectiva de género en el diseño de la cadena.

\subsection{Modelos de Madurez}

Para medir el grado de alineación podemos hacer uso de los modelos de madurez. La madurez se define como una medida para evaluar las capacidades de una organización en lo que respecta a una cierta disciplina (Cuenca et al. 2013). El principal valor de una evaluación de la madurez es captar la percepción de la propia empresa sobre la situación actual para apoyar el cambio y desarrollar una hoja de ruta para la mejora. Todos los modelos de madurez tienen unas características comunes: la definición de las áreas clave o dominios para ser evaluado y el nivel de madurez para cada área clave. El nivel de madurez representa un número de etapas acumulativas en las que las etapas superiores representan un nivel alto de madurez. Normalmente se trabaja con una escala Likert de 5 niveles.

Si hablamos de las propiedades de los modelos de madurez, (Magdaleno et al., 2011) definen una serie de propiedades que son comunes a todos los modelos de madurez:

a) El desarrollo de un único ámbito se simplifica y es descrito con número limitado de niveles de madurez.

b) Los niveles se caracterizan por tener ciertos requisitos.

c) Los niveles son acumulativos, esto quiere decir que los niveles más altos se construyen con la base de los 
niveles inmediatamente inferiores.

d) El número de niveles puede variar, pero están bien definidos y correctamente ordenados, desde niveles de una baja capacidad hasta un nivel final (la madurez)

e) Hay una progresión lógica entre los niveles y no se puede omitir ninguno de ellos.

f) Los niveles deben nombrarse con nombres cortos, lo que ayuda a dar una intención clara de cada nivel.

g) Las definici nes de cada nivel deben ser ampliadas para proporcionar un resumen de sus requisitos y medidas.

Con el objetivo de tratar de medir el grado de resiliencia y de agilidad de una CS, en la literatura se han identificado algunas aplicaciones de los modelos de madurez. El Marco de Evaluación de Madurez Adaptabilidad (RMAF)(Bhamidipaty, Lotlikar, and Banavar 2007) proporciona un enfoque holístico para evaluar los aspectos de la adaptabilidad organizacional. (Sanchis and Poler 2011) indican que se han definido métricas para evaluar la resiliencia empresarial, pero suel- en ser métricas relacionadas con la resiliencia una vez ocurrida la disrupción y que analizan lo rápido que la empresa se recupera y vuelve a su estado normal de operación. Sin embargo, el objetivo principal sería poder analizar lo resiliente y ágil que es una CS antes de que el evento disruptivo surja, para así poder, dependiendo de los resultados del análisis, tomar las medidas oportunas para mejorar su capacidad en este aspecto.

\section{Propuesta}

Tomando como referencia el informe de (Miles 2011) y (GRI \& IFC 2009) se propone la definición preliminar de un modelo de madurez que permita evaluar la incorporación de la perspectiva de género en las organizaciones sin limitar su agilidad y resiliencia. Las categorías identificadas en el informe, corresponderán con áreas clave del modelo de madurez propuesto que deberán ser evaluadas, se incluirán cinco niveles de alcance, no contemplados antes, y se incorporan los aspectos de agilidad y resiliencia que no habían sido considerados. Cada una de las áreas clave incluirá una serie de objetivos que deben ser alcanzados. El modelo propuesto se define en la Tabla 1.

Tabla 1Propuesta de modelo de madurez Integrado para la agilidad, resiliencia y perspectiva de género en las organizaciones (Desarrollado a partir de (Miles 2011).

\begin{tabular}{|c|c|c|c|c|}
\hline & \multicolumn{4}{|c|}{ Niveles de madurez } \\
\hline Áreas & 2 & 3 & 4 & 5 \\
\hline Gobierno y Valores & \multicolumn{4}{|c|}{$\begin{array}{c}\text { Objetivo: Promover la diversidad entre los miembros de la dirección, subrayando la importancia de conseguir la } \\
\text { igualdad de género, sin interferir en la agilidad y resiliencia de la organización. }\end{array}$} \\
\hline \multirow{4}{*}{ Ambiente de Trabajo } & \multicolumn{4}{|c|}{$\begin{array}{c}\text { Objetivo: Garantizar un ambiente de trabajo seguro y saludable para todos y todas, en un entorno ágil y resil- } \\
\text { iente. }\end{array}$} \\
\hline & \multicolumn{4}{|c|}{$\begin{array}{l}\text { Objetivo: Apoyar el desarrollo de los recursos humanos y promover acciones para generar oportunidades iguales } \\
\qquad \text { en el ambiente de trabajo ágil y resiliente. }\end{array}$} \\
\hline & \multicolumn{4}{|c|}{$\begin{array}{l}\text { Objetivo: Establecer de forma ágil la proporción de remuneración de todos los empleados y empleadas, por } \\
\text { categoría funcional y sin discriminar por género. }\end{array}$} \\
\hline & \multicolumn{4}{|c|}{$\begin{array}{l}\text { Objetivo: Tener unas condiciones laborales justas e imparciales, ofreciendo oportunidades iguales de trabajo } \\
\text { que puedan adaptarse a las necesidades cambiantes del entorno. }\end{array}$} \\
\hline \multirow{3}{*}{$\begin{array}{l}\text { Cadena de } \\
\text { suministro }\end{array}$} & \multicolumn{4}{|c|}{ Objetivo: Garantizar negocios ágiles y resilientes con organizaciones que respeten la igualdad de género. } \\
\hline & \multicolumn{4}{|c|}{ Objetivo: Promover de forma ágil la actitud emprendedora femenina. } \\
\hline & \multicolumn{4}{|c|}{$\begin{array}{l}\text { Objetivo: Divulgar informes sobre políticas de igualdad de género a todos los niveles de la cadena, y cómo estos } \\
\text { contribuyen a la agilidad y resiliencia de la misma. }\end{array}$} \\
\hline
\end{tabular}




\begin{tabular}{|c|c|}
\hline \multirow{3}{*}{ Comunidad } & $\begin{array}{l}\text { Objetivo: Definir acciones, incluyendo donaciones y subvenciones para abordar la igualdad en la comunidad ágil } \\
\text { y resiliente. }\end{array}$ \\
\hline & $\begin{array}{l}\text { Objetivo: Garantizar un número suficiente de programas, ágiles y resilientes, de participación/inversión en la } \\
\text { comunidad dirigidos a las mujeres. }\end{array}$ \\
\hline & $\begin{array}{c}\text { Objetivo: Contribuir al bienestar de las mujeres y hombres en las comunidades afectadas en un entorno cam- } \\
\text { biante. }\end{array}$ \\
\hline \multirow[t]{3}{*}{ Consumidores } & $\begin{array}{l}\text { Objetivo: Reflejar las necesidades de hombres y mujeres en los procesos y servicios de atención al cliente y en } \\
\text { los procedimientos de reclamación que se puedan dar bajo condiciones de incertidumbre }\end{array}$ \\
\hline & $\begin{array}{l}\text { Objetivo: No discriminar o estereotipar hombres o mujeres en las acciones de marketing y publicidad, sin per- } \\
\text { juicio de la agilidad y resiliencia. }\end{array}$ \\
\hline & $\begin{array}{c}\text { Objetivo: Abordar acciones desde gerencia para conseguir la "Certificación de Igualdad de Géneros" por ter- } \\
\text { ceros. }\end{array}$ \\
\hline \multirow[t]{2}{*}{ Inversión } & $\begin{array}{c}\text { Objetivo: Promover a las mujeres como inversoras, y la diversidad de los accionistas, para mejorar la agilidad y } \\
\text { resiliencia de la organización. }\end{array}$ \\
\hline & Objetivo: Ser reconocido por los inversores por el buen desempeño en cuanto a género, agilidad y resiliencia. \\
\hline
\end{tabular}

De manera general los niveles de 1 a 5 indicarán: El 1er nivel, Inicial, es el nivel más básico, representa una organización donde algunos criterios no se han analizado y nadie asume la responsabilidad de iniciar una discusión más estratégica. El $2^{\circ}$ nivel, Gestionado, se caracteriza por una organización que comienza a implementar acciones para alcanzar el objetivo marcado, sobre una base bien definida, pero dejando a la organización muy dependiente de la competencia de los gestores del sistema para cada criterio. El nivel $3^{\circ}$ se conoce como el nivel Establecido. Implica que la organización ha establecido un enfoque centralizado y coordinado para alcanzar el objetivo deseado. Esto generalmente se establecerá mediante el nombramiento de un administrador central que distribuirá procedimientos estándar. Este enfoque estandarizado es un requisito previo para poder implementar mejoras futuras en toda la organización. Una organización de nivel 3 también tiene roles y responsabilidades claramente definidos. El $4^{\circ}$ nivel, denominado Administrado, es similar al $3^{\circ}$, pero necesita que el enfoque coordinado centralmente esté apoyado por una mayor recopilación de datos. Requiere también mediciones regulares y pruebas con escenarios de uso que puedan hacerlo estadísticamente predecible y permitir mejoras estratégicas. El nivel de Mejora Continua, que es el $5^{\circ}$ y último nivel del modelo de madurez, es alcanzado por una organización que puede utilizar la retroalimentación obtenida de las prácticas del nivel 4 para mejorar continuamente.

Del modelo propuesto se pretende extraer la relación que indique qué aspectos de agilidad y resiliencia pueden verse afectados por las áreas donde la perspectiva de género debe ser incorporada.

Las actividades dirigidas a conseguir agilidad y resiliencia en la cadena de suministro tienen que incluir el requisito de incorporar y garantizar la equidad de género en la organización. Mientras que garantizar esta equidad, no puede limitar la capacidad de reacción. recuperación y flexibilidad de la organización

Se propone la relación entre los elementos a tener en cuenta en la incorporación de la perspectiva de género en las organizaciones, y los aspectos a tener en cuenta para garantizar su agilidad y resiliencia (Tabla 2 ). 
Tabla 2 Relación entre los elementos a tener en cuenta en la incorporación de la perspectiva de género junto a la agilidad y resiliencia en las organizaciones.

\begin{tabular}{|c|c|c|c|c|c|c|}
\hline & \multicolumn{6}{|c|}{ Agilidad y Resiliencia (Bhamidipaty, Lotlikar, and Banavar 2007) } \\
\hline $\begin{array}{l}\text { Perspectiva de } \\
\text { Género(GRI \& } \\
\text { IFC 2009) (Miles } \\
\text { 2011) }\end{array}$ & $\begin{array}{l}\text { Instalaciones y } \\
\text { recursos }\end{array}$ & Tecnología & $\begin{array}{l}\text { Datos y Aplica- } \\
\text { ciones }\end{array}$ & Procesos & Personas & Estrategia \\
\hline $\begin{array}{c}\text { Gobierno y } \\
\text { Valores }\end{array}$ & $\mathrm{x}$ & $x$ & $x$ & $x$ & $x$ & $x$ \\
\hline $\begin{array}{c}\text { Ambiente de } \\
\text { Trabajo }\end{array}$ & $x$ & & & $x$ & $x$ & $x$ \\
\hline $\begin{array}{l}\text { Cadena de } \\
\text { Suministro }\end{array}$ & & & & $x$ & $x$ & $x$ \\
\hline Comunidad & & & & $x$ & $x$ & $x$ \\
\hline Consumidores & & & & $x$ & $x$ & $x$ \\
\hline Inversión & & & & & $x$ & $x$ \\
\hline
\end{tabular}

El área clave Gobierno y valores implican establecer los principios que guíen las consideraciones sobre igualdad de género en políticas que determinan la cultura organizacional. Estos principios deben ser extendidos a todas las áreas incluidas como importantes para garantizar la agilidad y resiliencia de la organización. Ambiente de trabajo corresponde a la defi ición de oportunidades iguales para mujeres y hombres, eliminando aquellos obstáculos que limiten su participación. Lo que se relacionará con la definición de las instalaciones y recursos, procesos, personas y estrategia, que favorezca la agilidad y resiliencia. La CS corresponde a las nuevas oportunidades de empleo para las mujeres, tanto como empleadas como emprendedoras. Evaluar el desempeño de género en toda la CS puede ser una parte importante de la gestión de riesgo de las organizaciones y afecta principalmente a los procesos, personas y estrategias. La comunidad y los consumidores impactan en los procesos, las personas y la estrategia, al referirse a fortalecer los esfuerzos para promover la igualdad de géneros en la organización, implementar la concienciación de género en la atención al cliente, y analizar el feedback de los clientes, así como, desarrollar un grupo más inclusivo de contratación dentro de la comunidad. Por último respecto al área clave inversión, se centra principalmente en las decisiones de los inversores que son frecuentemente tomadas basadas en aspectos de interés de su desempeño en lo que se refiere al género, lo que corresponde con las áreas de personas y estrategia.

\section{Conclusiones}

La igualdad es un derecho recogido en nuestra Constitución. Incorporar la perspectiva de género se está convirtiendo en un requisito legal obligatorio para las organizaciones con el fin de garantizar dicho derecho. Por otra parte, el entorno cambiante al que se enfrentan las empresas les obliga a ser ágiles y resilientes para recuperarse ante eventos externos e internos que puedan producirse. Las actividades dirigidas a conseguir esta agilidad y resiliencia, no pueden dejar de lado el requisito de incorporar y garantizar la equidad de género en la organización. Así mismo, garantizar esta equidad de género no debe poner en riesgo la capacidad de recuperación de la organización. Para tener en cuenta estos aspectos, la propuesta aquí presentada identifica y relaciona las áreas clave a considerar respecto a agilidad, resiliencia y equidad de género. De este modo, ayudará a las empresas a incorporarlas en la definició de las organizaciones. Como líneas futuras se propone completar el modelo detallando qué implica estar en cada nivel de madurez para cada una de las áreas clave a evaluar y su aplicación sobre una cadena de suministro. 


\section{Referencias}

Alesina L. (2007) Guía metodológica para el estudio de cadenas productivas con perspectiva de género. Biblioteca Asocam, , http://asocam.org/biblioteca/ items/show/1603.

Australian Goverment. 2016. 'The Business Case for Gender Equality.' https://goo.gl/kc2CEQ

Avison, David, David Wilson, Jill Jones, and Philip Powell. 2004. 'Using and Validating the Strategic Alignment Model.' The Journal of Strategic Information Systems 13(3): 223.

Bhamidipaty, Anuradha, Rohit Lotlikar, and Guruduth Banavar. 2007. 'RMI: A Framework for Modeling and Evaluating the Resiliency Maturity of IT Service Organizations.' In IEEE International Conference on Services Computing (SCC 2007), IEEE, 300-307.

Boza, Andres, María Del Mar Alemany Díaz, Eduardo Vicens Salort, and L. Cuenca. 2014. 'Event Management in Decision-Making Processes with Decision Support Systems.' Agora University Editing House. http://hdl.handle.net/10251/71711.

Caven, Valerie, Elena Navarro-Astor, and Marie Diop. 2016. 'A Cross-National Study of Gender Diversity Initiatives in Architecture' ed. Alain Klarsfeld, Eddy S.W. Ng, Liza Castr. Cross Cultural \& Strategic Management 23(3): 431-49.

Cinco Días. 2016. 'Las Empresas Frente a Las Amenazas Externas.' https://goo.gl/tU6GqU

Claire Aanes, Autores et al. 2013. 'Preguntas Frecuentes Sobre El Análisis de Género.' https://www. giz.de/expertise/downloads/ES-FAQ_analisis_de_ genero.pdf

Cuenca L., J. Milla, A.Boza 2015 Análisis de la alineación de las tecnologías de la información y el negocio. en empresas de la Comunidad ValencianaDirección y Organización 55 (2015) 38-43

Cuenca, L., A. Boza, M.M.E. Alemany, and Trienekens Jos J. 2013. 'Structural Elements of Coordination Mechanisms in Collaborative Planning Processes and Their Assessment through Maturity Models: Application to a Ceramic Tile Company.' Computers in Industry 64(8): 898-911.
Cuenca, L., A. Boza, and A. Ortiz. 2011. 'An Enterprise Engineering Approach for the Alignment of Business and Information Technology Strategy.' International Journal of Computer Integrated Manufacturing 24(11): 974-92.

GRI \& IFC. 2009. 'Embedding Gender in Sustainability Reporting A Practitioner's Guide.' https:// www.globalreporting.org/resourcelibrary/Embedding-Gender-In-Sustainability-Reporting.pdf

Haes, S De, and W Van Grembergen. 2004. 'IT Governance and Its Mechanisms.' Information Systems Control Journal 1, 27-33

Luftman, J. 2003. 'Assessing IT/business Alignment.' Information strategy 20(1): 7.Magdaleno, A. M., Araujo, R. M. De, \& Werner, C. M. L. (2011). A roadmap to the Collaboration Maturity Model (CollabMM) evolution. Proceedings of the 2011 15th International Conference on Computer Supported Cooperative Work in Design, CSCWD 2011, 105-112. https://doi. org/10.1109/CSCWD.2011.5960062

Miles, Katherine. 2011. 'Sustainability Accounting, Management and Policy Journal Embedding Gender in Sustainability Reports.' Journal Journal Iss Journal Iss 2(1): 139-46.

ONU Mujeres, \& Enciso, R. (2016). Principios para el empoderamiento de las mujeres en las empresas. Retrieved from http://www.onu.org.mx/wpcontent/ uploads/2016/12/Empoderamiento-mujeres-en-empresas.pdf

Oxfam. 2013. Mars, Mondelez Y Nestle Y La Lucha Por Los Derechos de Las Mujeres. http://imagenes.publico.es/resources/archivos/2013/3/5/1362507784493informe\%20oxfam.pdf

Prieto-Carron, M. (2008). Women, workers, industrialization, global supply chains and cor-porate codes of conduct. Journal of Business Ethics, 83(1), 5-17.

Sanchis, Raquel, and Raúl Poler. 2011. 'Medición de La Resiliencia Empresarial Ante Eventos Disruptivos. Una Revisión Del Estado Del Arte.' http://adingor.es/congresos/web/uploads/cio/cio2011/administracion_de_empresas/104-113.pdf 
Sanchis, R.; Poler, R. (2014). Enterprise resilience assessment: a categorisation framework of disruptions. Dirección y Organización. 54:45-53. http://hdl. handle.net/10251/58456.

Sheffi, Yossi. 2004. 'Demand Variability and Supply Chain Flexibility.' In Entwicklungspfade Und Meilensteine Moderner Logistik, Wiesbaden: Gabler Verlag, 85-117.
Soni, Umang, and Vipul Jain. 2011. 'Minimizing the Vulnerabilities of Supply Chain: A New Framework for Enhancing the Resilience.' In 2011 IEEE International Conference on Industrial Engineering and Engineering Management, IEEE, 933-39. 